\title{
NILPOTENCY IN ENDOMORPHISM RINGS
}

\section{ROBERT GORDON}

ABSTRACT. Nil subrings of the endomorphism ring of a module with finite Krull dimension sequence are nilpotent. This includes the case of a module with finite Krull dimension as well as noetherian modules. The method used is to embed the endomorphism ring, modulo a nilpotent ideal, in the endomorphism ring of an artinian object of a Grothendieck category.

The classical results, that nil subrings of artinian rings and of noetherian rings are nilpotent, have proved to be extendible to endomorphism rings of modules which are artinian or noetherian. Recently it has been proved [4], [6] that nil subrings of any ring with Krull dimension are nilpotent. Since this result includes the classical ones cited, the question arises whether nil subrings of the endomorphism ring of any module with Krull dimension are nilpotent. This article, by applying a heretofore unexploited technique to the known artinian case (due to Fisher [1]), provides an affirmative answer when the Krull dimension is finite.

We will use the concepts of [4] freely and denote the Krull dimension of $A \in / A=\bmod R, R$ a ring, by $K \operatorname{dim} A$. By $\tau_{a}^{\prime} A$ we mean the largest submodule of $A$ in the smallest localizing subcategory $\left\langle/ A_{\alpha}^{\prime}\right\rangle$ of $/ A$ containing the Serre subcategory $/ A_{\alpha}^{\prime}$ of modules of Krull dimension $<\alpha$. If $K \operatorname{dim} A$ exists we call the sequence of ordinals $-1=a_{0}<a_{1}<\cdots$ defined for $m \geq 0$ by

$$
\alpha_{m+1}=\min \left\{\alpha \mid \tau_{\alpha+1}^{\prime}\left(A / \tau_{\alpha_{m+1}}^{\prime} A\right) \neq 0\right\}
$$

the Krull dimension sequence of $A$. It can be seen, using [4, Theorem 4.1] and [5, Theorem 2.4], that the Krull dimension sequence of $A$ is finite if and only if no submodule of $A$ has limit ordinal Gabriel dimension-see [5].

Presented to the Society, January 18, 1974; received by the editors August 11, 1973.

AMS (MOS) subject classifications (1970). Primary 16A22, 16A64; Secondary $18 \mathrm{E} 15,18 \mathrm{E} 35$.

Key words and phrases. Module with Krull dimension, Krull dimension sequence, endomorph ism ring, nil ring, nilpotent ring, artinian object, Grothendieck category, noetherian module, noetherian ring, ring of quotients. 
Theorem 1. If the Krull dimension sequence of a module A with Krull dimension is finite then, modulo some nilpotent ideal, the endomorphism ring of $A$ embeds in the endomorphism ring of an artinian object of a Grothendieck category.

Proof. Let $-1=a_{0}<\alpha_{1}<\cdots<a_{r}$ be the Krull dimension sequence of $A$ and let $A_{m}=\tau_{a_{m}+1}^{\prime} A$. Since each $A_{m}$ is an invariant submodule of $A$, $B_{m}=A_{m} / A_{m-1}$ is canonically an $S=$ End $A$-module and restriction to $A_{m}$ induces an embedding of $S / H_{m}$ in End $B_{m}$, where $H_{m}=$ ann $_{s} B_{m}$. Let $H=$

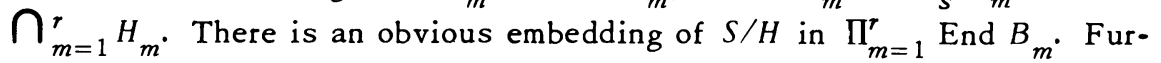
thermore, $H$ is nilpotent, for $H^{r} A \subseteq H_{1} \cdots H_{r-1} H_{r} A=0$ and $A$ is a faithful $S$-module.

Let $T_{m}$ be the canonical functor from $/ A$ to the quotient category $/ A /\left\langle/ A_{\alpha_{m}}^{\prime}\right\rangle$. If $T_{m} f=0$ for $f \in$ End $B_{m}$ then im $f \in\left\langle/ A_{\alpha_{m}}^{\prime}\right\rangle$ and the choice of $\alpha_{m}$ shows that $f=0$. Thus $T_{m}$ induces a monomorphism End $B_{m}$ $\rightarrow$ End $T_{m} B_{m}$. But by [4, Theorem 4.1] $K \operatorname{dim} B_{m}=\alpha_{m}$ and so, by [5, Proposition 1.5], $B_{m^{\prime}}$ as an object of $/ A / / A_{a_{m}}^{\prime}$, is artinian. Hence $T_{m} B_{m}$ is an artinian object of $/ A /\left\langle/ A_{\alpha_{m}^{\prime}}^{\prime}\right\rangle$, since this category is a quotient category of $/ A / / A_{a_{m}}^{\prime}$. Thus we have an embedding of $\Pi_{m=1}^{r}$ End $B_{m}$ in the endomorphism ring of the artinian object $\left(T_{1} B_{1}, \cdots, T_{r} B_{r}\right)$ of the Grothendieck category $/ A /\left\langle/ A_{a_{1}}^{\prime}\right\rangle \times \cdots \times / A /\left\langle/ A_{a_{r}}^{\prime}\right\rangle$.

Noting that Fisher's proof of his result mentioned above is valid for an artinian object of a Grothendieck category, we have

Theorem 2. If $A$ is a module with Krull dimension whose Krull dimension sequence is finite then each nil subring of End $A$ is nilpotent.

Corollary 3. Nil subrings of the endomorphism ring of a module with finite Krull dimension are nilpotent.

It is open whether nil subrings of the endomorphism ring of an arbitrary module with Krull dimension are nilpotent, and clearly there are major obstructions to extending the proof of Theorem 1. However, if the module $A$ in that theorem is noetherian, then the given proof becomes more elementary, and the rings End $T_{m}{ }_{m}$ are even semiprimary. In particular, we obtain

Proposition 4. Modulo a nilpotent ideal, the endomorphism ring of a noetherian module embeds in a semisimple artinian ring.

Of course this implies that nil subrings of such endomorphism rings are nilpotent, a result announced by Goldie and Small (see [3]). I do not 
know whether the nilpotent ideal of the proposition can be taken to be the prime radical; but there is an example [4, Example 6.9] which shows that the embedding obtained is not the embedding of a right order in its right quotient ring.

We end with an application of Proposition 4. Let $\mid B$ be a localizing subcategory of $/ A$, regard $/ A / \mid B$ as a full subcategory of $/ A$ and let $T$ : $/ A \rightarrow / A / \mid B$ be the canonical functor. If $A \in / A$ is noetherian then $T A$ is a noetherian object of $/ A / \mid B$ and we can deduce that nil subrings of End ${ }_{R} T A$ are nilpotent. In particular, since End $_{R} T A$ is easily seen to be isomorphic to the ring of quotients of $R$ with respect to $\mid B$, we have

Corollary 5. Nil subrings of any ring of quotients of a right noetherian ring are nilpotent.

We remark that the same is true of a ring with finite Krull dimension. We also note, vis-à-vis Proposition 4, that Feller and Deshpande [2, Proposition 3.2] have stated that nil subrings of the endomorphism ring of the rational completion of a noetherian module are nilpotent. Although there was a gap in their proof (subsequently filled), the result is valid. It is just the special case of the above discussion when $\mid B=\{C \in / A \mid \operatorname{Hom}(C, E(A))=0\}, E(A)$ the injective envelope of $A$.

Acknowledgement. I would like to thank Professor I. N. Herstein for inviting me to the 1973 Ring Theory Conference at the University of Chicago, during which this paper was written.

\section{REFERENCES}

1. J. W. Fisher, Nil subrings of endomorphism rings of modules, Proc. Amer. Math. Soc. 34(1972), 75-78. MR 45 \#1960.

2. E. H. Feller and M. G. Deshpande, Endomorphism ring of essential extension of a noetherian module, 1973 (preprint).

3. A. W. Goldie and L. W. Small, A note on rings of endomorphisms, J. Algebra 24 (1973), 392-395.

4. R. Gordon and J. C. Robson, Krull dimension, Mem. Amer. Math. Soc. No. 133(1973).

5. - The Gabriel dimension of a module, J. Algebra (to appear).

6. T. H. Lenagan, The nil radical of a ring with Krull dimension (to appear).

DEPARTMENT OF MATHEMATICS, TEMPLE UNIVERSITY, PHIL ADELPHIA, PENNSYLVANIA 19122 